greater cytoplasmic translocation of HMGB1. These changes were most marked in regions distal to active lesional areas ('active lesion' tissue blocks) but were also noted in the majority of tissue blocks without an active lesion ('NAWM' tissue block). Both chronic white matter and cortical demyelinating regions demonstrated reduced expression of HMGB1.

Conclusion Increased expression of HMGB1 in CNS-resident cells supports the notion of pervasive tissue alteration in MS brain tissue. Whether this expression pattern reflects a pro-inflammatory, destructive process or a protective response to surrounding cellular stress or indeed a balance between the two, is yet to be determined.

\section{HIGH-MOBILITY GROUP BOX 1 (HMGB1) EXPRESSION IS INCREASED IN THE NORMAL-APPEARING BRAIN TISSUE OF MULTIPLE SCLEROSIS (MS) PATIENTS VS. CONTROLS}

Ghaniah Z Hassan-Smith, ${ }^{1,2}$ Souad Messahel, ${ }^{1}$ Gordon Mazibrada, ${ }^{2}$ Ana Maria Gonzalez, ${ }^{1}$ Santhosh Nagaraju, ${ }^{2}$ Martyn Carey, ${ }^{2}$ John Curnow, ${ }^{1}$ Michael Douglas ${ }^{1,3}$. 'University of Birmingham; ${ }^{2}$ Queen Elizabeth Hospital, University Hospitals Birmingham NHS Foundation Trust; ${ }^{3}$ Dudley Group NHS Foundation Trust, Dudley

\subsection{6/jnnp-2014-309236.133}

Background MS is characterised pathologically by focal inflammatory, demyelinating plaques. More recently, normal-appearing white and grey matter (NAWM/ NAGM) distal to these lesions have also been shown to be altered in MS, suggesting more widespread changes affecting MS brain tissue. High-mobility group box 1 (HMGB1) acts as a damage-associated pattern recognition molecule (DAMP) released by activated macrophages, in addition to sensing sterile damage and propagating subsequent inflammatory responses. Whilst increased macrophage/microglial HMGB1 expression has been demonstrated previously in active lesions, we extended these findings, including analysis of non-lesional brain tissue in MS patients vs. non-MS controls.

Results Semi-quantitative immunoreactivity (IR) analysis showed increased HMGB1 expression in active WM lesions in MS vs. control WM tissue, predominantly affecting macrophages/ activated microglial cells. However, IR was also significantly increased in non-lesional brain tissue in MS patients vs. controls, with the changes consistently marked in oligodendroglial cells in both grey and white matter. In addition, neuronal cells in non-lesional cortical regions demonstrated significantly 